\title{
Schwarzschild black branes and strings in higher-dimensional brane worlds
}

\author{
P. Kanti \\ CERN, Theory Division, CH-1211 Geneva 23, Switzerland \\ I. Olasagasti and K. Tamvakis \\ Physics Department, University of Ioannina, GR-451 10 Ioannina, Greece
}

(Received 6 August 2002; published 27 November 2002)

\begin{abstract}
We consider branes embedded in spacetimes of codimension 1 and 2, with a warped metric tensor for the subspace parallel to the brane. We study a variety of brane-world solutions arising by introducing a Schwarzschild-like black hole metric on the brane and we investigate the properties of the corresponding higher-dimensional spacetime. We demonstrate that normalizable bulk modes lead to a vanishing flow of energy through the naked singularities. From this point of view, these singularities are harmless.
\end{abstract}

DOI: 10.1103/PhysRevD.66.104026

PACS number(s): 04.50.+h, 04.70.Bw, 11.10.Kk, 11.25.Mj

\section{INTRODUCTION}

Higher-dimensional models of gravitation are motivated by the need to explain the large difference in magnitude between two important energy scales in nature: the Planck scale $M_{P} \sim 2 \times 10^{18} \mathrm{GeV}$ that governs the gravitational interactions, and the electroweak scale of particle physics. The study of these models during the past few years has led to a number of interesting theoretical ideas [1-3], among them the possibility of extra noncompact dimensions [3] and the effective localization of gravity. In the latter case, the higherdimensional spacetime is filled with a negative cosmological constant that gives rise to an AdS spacetime. The standard model interactions are confined on zero-thickness 3-branes, while the graviton can propagate in the five-dimensional bulk. Four-dimensional gravity is successfully reproduced on the brane by an ordinary massless graviton localized on it [4], while the four-dimensional Planck mass is merely an effective scale derived from the fundamental energy scale and the AdS radius of the five-dimensional spacetime.

In a five-dimensional framework, such as the RandallSundrum (RS) model, it would be natural to expect that when matter, trapped on the brane, undergoes gravitational collapse, the four-dimensional background that forms would be described by a Schwarzschild-type metric. The first attempt to substitute the flat, Minkowski background on the brane by a Schwarzschild spacetime, in the context of the one-brane RS model, led to the derivation of the RSSchwarzschild black-hole solution [5]. The induced metric on the brane comes out to be purely a Schwarzschild metric, as expected, respecting all the usual astrophysical constraints [6]. However, the solution, when seen from the fivedimensional point of view, describes a black string, an extended black-hole singularity, infinite in the fifth dimension. As the study of the curvature invariant quantities of the theory reveal, the AdS horizon, located at infinity, has also been changed to a true, naked singularity of the fivedimensional spacetime. A localized black cigar solution, with a finite extension along the fifth dimension, might arise due to a Gregory-Laflamme type of instability [7] that appears near the AdS horizon.

The subject of black holes in the context of extra dimen- sions has been extensively studied during the past few years [8-13]. In a recent paper [14], two of us considered fourdimensional metric Ansätze of the Schwarzschild type with a horizon depending on the fifth dimension aiming at finding localized black-hole solutions. Nevertheless, such configurations turned out to be compatible only with exotic bulkmatter distributions. Although in these solutions the harmless character of the AdS horizon was restored and the black-hole singularity rapidly "decayed" away from the brane, the model was infested with an additional singularity located at the induced horizon of the black hole.

It has become clear by now that the embedding of a fourdimensional black hole into a higher-dimensional spacetime leads to the change of the topological properties of the $d$-dimensional spacetime in a dramatic way: the fourdimensional black-hole singularity becomes manifest also in the higher-dimensional curvature invariants, while previously harmless $d$-dimensional horizons now become true spacetime singularities. In the present paper, we consider models of maximum codimension 2 , with a factorizable metric for the subspace parallel to the brane. We perform a singularity analysis that tries to shed light on the topological structure of various five-, six-, or higher-dimensional spacetimes of the particular type, and we examine the inevitable appearance of black-hole and naked singularities in the square of the Riemann tensor. Whenever necessary, the naked singularity is shielded by a second brane, which automatically leads to a finite-size black tube construction that interpolates between two four-dimensional Schwarzschildlike black holes. In solutions, however, with a cosh-like profile for the warp factor, along one or more extra dimensions, an effective localization of both the black string and the bulk singularity may take place through the suppression of the singular terms, appearing in the curvature invariants, away for the brane. In the context of single-brane configurations, the bulk singularity may terminate the infinitely extended black string, however a nonvanishing flow of energy through the naked singularities of the spacetime would lead to the breakdown of the conservation of currents associated with spacetime symmetries. We therefore examine whether normalizable bulk modes lead to a vanishing flow of energy and 
render the naked singularities harmless from this point of view.

The outline of this paper is as follows. Section II presents our spacetime Ansätz and useful formulas to be used in the following analysis. In Sec. III, we focus on the study of five-dimensional brane-world solutions and we consider three categories depending on the profile of the warp factor along the extra dimension. Section III extends our analysis in higher-dimensional models and we study a variant of the Cohen-Kaplan [15] global string model, which has cylindrical symmetry in the transverse space, and a $d$-dimensional version of the cylindrically nonsymmetric model of Kanti, Madden, and Olive [16] (KMO). We present our conclusions in Sec. V.

\section{MODELS OF GENERAL CODIMENSION}

In what follows, ${ }^{1}$ we are going to consider warped geometries of codimension higher than 1 . The total number of dimensions $d$ is the sum of $q$ longitudinal dimensions along the brane (occasionally we will specialize in the case $q$ $=4$ ) and $n$ transverse dimensions. By warped geometries we mean all those with line elements that can be written as

$$
\begin{aligned}
d s^{2} & =e^{2 A(y)} d \hat{s}_{q}^{2}+d \widetilde{s}_{n}^{2} \\
& \equiv e^{2 A(y)} \hat{g}_{\mu \nu}(x) d x^{\mu} d x^{\nu}+\gamma_{a b}(y) d y^{a} d y^{b}
\end{aligned}
$$

In the above metric, $d \hat{s}^{2}$ is a $q$-dimensional line element that depends only on the longitudinal coordinates $\left\{x^{\mu}\right\}$. Likewise, $d \widetilde{s}_{n}^{2}$ refers to an $n$-dimensional line element that depends only on the transverse coordinates $\left\{y^{a}\right\}$. Henceforth, we will be using a hat and a tilde in order to denote quantities evaluated exclusively in terms of the $\hat{g}_{\mu \nu}$ and $\gamma_{a b}$ metric tensors, respectively.

For the above general warped metric, the various components of the Ricci tensor are

$$
\begin{aligned}
& R_{a b}=\widetilde{R}_{a b}-q\left(\tilde{\nabla}_{a} \tilde{\nabla}_{b} A+\tilde{\nabla}_{a} A \tilde{\nabla}_{b} A\right), \\
& R_{\mu \nu}=\hat{R}_{\mu \nu}-\hat{g}_{\mu \nu} e^{2 A}\left[\tilde{\nabla}^{2} A+q(\tilde{\nabla} A)^{2}\right], \\
& R_{a \mu}=0 .
\end{aligned}
$$

The resulting Ricci scalar is

$$
R=\widetilde{R}_{(n)}+\hat{R}_{(q)} e^{-2 A}-2 q\left(\tilde{\nabla}^{2} A\right)-q(q+1)(\tilde{\nabla} A)^{2} .
$$

\footnotetext{
${ }^{1}$ We consider the standard Einstein gravitational action

$$
\begin{aligned}
\mathcal{S}= & \int d^{d} X \sqrt{-G}\left\{2 M^{d-2} R-\Lambda_{B}\right\} \\
& -\int d^{d} X \sqrt{-G} \mathcal{L}-\int d^{(d-1)} x \sqrt{-h} \hat{\mathcal{L}},
\end{aligned}
$$
}

where the last term corresponds to a localized source on a wall with induced metric $h_{a b}$.
Another quantity of special interest is the square of the Riemann tensor, ${ }^{2}$ which reveals, more accurately than any other scalar quantity, the singular properties of spacetimes containing black-hole line elements. It can be computed to be

$$
\begin{aligned}
\left(R_{B C D}^{A}\right)^{2}= & \left(\widetilde{R}_{b c d}^{a}\right)^{2}+e^{-4 A}\left(\hat{R}_{\mu \nu \lambda}^{\alpha}\right)^{2}-4 e^{-2 A}(\widetilde{\nabla} A)^{2} \hat{R}_{(q)} \\
& +4 q\left(\widetilde{\nabla}_{a} \widetilde{\nabla}_{b} A+\widetilde{\nabla}_{a} A \widetilde{\nabla}_{b} A\right)^{2} \\
& +2 q(q-1)(\tilde{\nabla} A)^{2}(\tilde{\nabla} A)^{2}
\end{aligned}
$$

From the Einstein tensor for the warped metrics, we can read out the structure of energy-momentum tensors compatible with it:

$$
\begin{aligned}
T_{a b} & =\widetilde{T}_{a b}(y)-\frac{1}{2} \gamma_{a b}(y) e^{-2 A(y)} \hat{R}_{(q)}(x), \\
T_{\mu \nu} & =\hat{T}_{\mu \nu}(x)+\hat{g}_{\mu \nu}(x) \Delta(y), \\
T_{a \mu} & =0 .
\end{aligned}
$$

$T_{a b}$ depends on $\left\{x^{\mu}\right\}$ only through $\hat{R}_{(q)}(x)$, the Ricci scalar of $d \hat{s}_{q}^{2} \cdot T_{\mu \nu}$, on the other hand, depends on $\left\{y^{a}\right\}$ only through a term proportional to $\hat{g}_{\mu \nu}(x), \Delta(y)$, which is not universal but depends on the particular source in the bulk.

For metrics such as Eq. (2.1), the equation for $\hat{g}_{\mu \nu}$ decouples. In fact, one can show that the Einstein tensor for $\hat{g}_{\mu \nu}$ is

$$
\hat{G}_{\mu \nu}=\hat{T}_{\mu \nu}-K \hat{g}_{\mu \nu},
$$

where $\hat{T}_{\mu \nu}$ is the $x^{\mu}$-dependent part of the energy-momentum tensor and what appears here as a cosmological constant term $K$ is just a separation constant. This means that as long as we fix $K$, independently of the explicit form of the 4D solution, the geometry along the transverse space and the warp factor will remain the same. This is true in particular for all the solutions with $\hat{T}_{\mu \nu}=0$. There are many examples of this class in the literature [17]. For the most part, the existing solutions consider the maximally symmetric 4D solutions. It is clear, however, from the discussion above, that we can generalize any of them by considering the corresponding black-hole solution counterparts. This will work both for Schwarzschild and Kerr black holes but not for charged ones since the energy-momentum tensor for the charged black hole on the brane does not fall into the class described by the above warped geometries [18].

$$
\begin{aligned}
& { }^{2} \text { The Riemann tensor components different from zero are } R_{b c d}^{a} \\
& =\widetilde{R}_{b c d}^{a} \text { and } \\
& \qquad \begin{aligned}
R_{b \nu d}^{\alpha} & =-\delta_{\nu}^{\alpha}\left(\widetilde{\nabla}_{b} \widetilde{\nabla}_{d} A+\widetilde{\nabla}_{b} A \widetilde{\nabla}_{d} A\right), \\
R_{\mu \nu d}^{a} & =g_{\mu \nu}\left(\widetilde{\nabla}_{d} \tilde{\nabla}^{a} A+\tilde{\nabla}^{a} A \widetilde{\nabla}_{d} A\right), \\
R_{\mu \nu \lambda}^{\alpha} & =\hat{R}_{\mu \nu \lambda}^{\alpha}+(\widetilde{\nabla} A)^{2}\left(g_{\mu \nu} \delta_{\lambda}^{\alpha}-g_{\mu \lambda} \delta_{\nu}^{\alpha}\right) .
\end{aligned}
\end{aligned}
$$


An important thing to note is that, for any nontrivial 4D geometry, any zero of the warp factor will give rise to a naked singularity. We can see this from the expressions (2.5) and (2.6). If either $\left(\hat{R}_{\mu \nu \lambda}^{\alpha}\right)^{2}$ or $\hat{R}_{(q)}$ is nonzero due to a nontrivial $x^{\mu}$ dependence, a singularity will develop wherever the warp factor goes to zero.

A naked singularity could be tolerated and considered harmless if no conserved quantities flow into the singularity. If $K^{A}$ is a Killing vector, this will give rise to an associated conserved current $J^{A}=T_{B}^{A} K^{B}$. If the horizon has a normal vector $n^{A}$ and we define $z$ locally by $d z=n^{A} d X_{A}$, then the flow of the conserved current into the singularity, at $z=0$, is given by

$$
\lim _{z \rightarrow 0} \int_{z} n_{A} J^{A} d \sigma
$$

where $d \sigma$ is the invariant volume element of the constant $z$ hypersurfaces. So, the condition for vanishing flow is

$$
\lim _{z \rightarrow 0} n_{A} J^{A} d \sigma=\lim _{z \rightarrow 0} n_{A} T_{B}^{A} K^{B} d \sigma=0 .
$$

For a scalar field $\phi(x, y)$ propagating in the bulk, the condition of vanishing flow reduces to

$$
\lim _{z \rightarrow 0} \partial_{z} \phi K^{A} \partial_{A} \phi d \sigma=0 .
$$

In the following sections, we will apply the above scheme on five-, six, or higher-dimensional models and look for scalar modes that respect the above constraint and ensure vanishing flow of conserved currents through the naked singularities of the spacetime.

\section{THE FIVE-DIMENSIONAL CASE}

In this section, we focus on the case of a five-dimensional spacetime, whose line element may be written in the factorized form

$$
d s^{2}=g_{M N} d x^{M} d x^{N}=e^{2 A(y)} \hat{g}_{\mu \nu}(x) d x^{\mu} d x^{\nu}+d y^{2} .
$$

The four-dimensional metric, $\hat{g}_{\mu \nu}$, is assumed to describe a spherically symmetric black-hole background. As mentioned in the Introduction, we expect that the embedding of the four-dimensional black hole into the five-dimensional spacetime will lead to the manifestation of the $4 \mathrm{D}$ black-hole singularity in the five-dimensional curvature invariants. Moreover, five-dimensional horizons located at the boundaries of the extra dimension will now become true spacetime singularities. In this section, we perform, first, a singularity analysis that tries to shed light on the topological structure of various five-dimensional spacetimes of the type (3.1), and secondly, a study of the possible flow of energy through the true singularities of the spacetime.

Starting from the Ansätz (3.1), we now consider three different cases of five-dimensional spacetimes, defined by the behavior of the warp factor $e^{A(y)}$ along the extra dimension.

\section{A. Asymptotically vanishing warp factor}

In this category belongs the class of line elements that are characterized by the vanishing of the warp factor $e^{A(y)}$ at an infinite coordinate distance from the brane. A typical example of this type is the spacetime describing the fivedimensional black string solution [5] with line element

$$
\begin{aligned}
d s^{2}= & e^{-2 k|y|}\left\{-\left(1-\frac{2 M}{r}\right) d t^{2}+\left(1-\frac{2 M}{r}\right)^{-1} d r^{2}\right. \\
& \left.+r^{2}\left(d \theta^{2}+\sin ^{2} \theta d \varphi^{2}\right)\right\}+d y^{2},
\end{aligned}
$$

where $k^{2}=\kappa_{5}^{2}\left|\Lambda_{B}\right| / 6$. In this case, the five-dimensional bulk is filled with a negative cosmological constant $\Lambda_{B}$, and the line element remains invariant under the mirror transformation $y \leftrightarrow-y$, with the infinitely thin, positive-tension brane located at $y=0$. By making use of Eq. (2.6), the Riemann curvature invariant for the above background comes out to be

$$
R_{A B C D} R^{A B C D}=40 k^{4}+\frac{48 M^{2} e^{4 k|y|}}{r^{6}}
$$

and it clearly diverges at the black-hole singularity at $r=0$, and the AdS horizon $|y| \rightarrow \infty$. For factorized fourdimensional line elements like the one above, the only method that makes the AdS singularity disappear and gives a finite size to the black-hole singularity is the introduction of a second, negative-tension brane at $y=L<\infty$. The induced metric tensor on both branes will describe a four-dimensional black hole. If we choose the inter-brane distance to be the one that resolves the hierarchy problem [2], then a black tube construction, extending into the bulk, will interpolate between two brane black holes whose masses, and horizon values, will differ by 16 orders of magnitude.

It is nevertheless interesting to investigate whether we can have a nonvanishing flow of energy into the bulk singularities in the case of the single-brane configurations, when the second brane is absent. Here, we follow the method of Ref. [15], however our analysis considerably deviates from theirs at certain points due to the different metric Ansätz. The equation of a massless scalar field propagating in the background (3.1) may be written as

$$
e^{-2 A} \partial_{y}\left(e^{4 A} \partial_{y} \Phi_{y}\right)+m^{2} \Phi_{y}=0,
$$

under the assumption that the scalar field has the factorized form $\Phi(x, y)=\Phi_{x}(x) \Phi_{y}(y)$, and

$$
\frac{1}{\sqrt{-\hat{g}}} \partial_{\mu}\left[\sqrt{-\hat{g}} \hat{g}^{\mu \nu} \partial_{\nu} \Phi_{x}\right]=m^{2} \Phi_{x} .
$$

We first look for solutions to the above equation (3.4) describing massless modes, $m^{2}=0$. Then, Eq. (3.4) clearly reveals the existence of two zero-mode solutions: 


$$
\Phi_{y}^{(1)}=\mathrm{const}, \quad \Phi_{y}^{(2)}=\mathrm{const} \int^{y} \frac{d y^{\prime}}{e^{4 A\left(y^{\prime}\right)}} .
$$

The metric Ansätz (3.2) is characterized by translation invariance along the $(t, \theta, \varphi)$ coordinates and rotational invariance in the four-dimensional spacetime. For our purposes, it is enough to consider the Killing vector $K_{M}^{(\mu)}=e^{2 A} \delta_{M}^{\mu}$, with $\mu=t, \theta, \varphi$, and demand that no flow of energy, associated with the current $J_{(\mu)}^{y}=T^{y N} K_{N}^{(\mu)}$, takes place at the location of the bulk singularity. That means we should have

$$
\begin{aligned}
\lim _{|y| \rightarrow \infty} & \sqrt{-g} J_{(\mu)}^{y} \\
\quad & =\lim _{|y| \rightarrow \infty}\left[\sqrt{-\hat{g}} \hat{g}^{\mu \nu} e^{4 A} \Phi_{x}\left(\partial_{x} \Phi_{x}\right) \Phi_{y}\left(\partial_{y} \Phi_{y}\right)\right]=0 .
\end{aligned}
$$

Taking into account the behavior of the two solutions (3.6) near the AdS horizon, $\Phi_{y}^{(1)}=$ const and $\Phi_{y}^{(2)}=$ const $e^{4 k|y|}$, we may see that the flow of energy is indeed zero for the first solution while it diverges for the second one. We need, therefore, to discard the latter as an irregular solution and keep only the former as the physically acceptable, regular solution. As a result, for the massless modes, it is only the scalar configurations with a trivial profile along the extra dimension that are acceptable.

We may easily check that, under the assumption that $\Phi_{y}$ = const, all the remaining components of the current $J^{M}$ also vanish at the AdS boundary. We should stress at this point that the four-dimensional line element has a nontrivial topology that introduces an extra singularity, the black-hole one, in the five-dimensional spacetime. This singularity is protected by a horizon and thus it is harmless. As for all scalar modes propagating in a black-hole background, we demand that they are well defined in the regime $r>r_{H}=2 M$ and have a finite value at the black-hole horizon.

We now turn to the massive scalar modes. By performing a coordinate transformation $d y=e^{A} d z$, and going to conformal coordinates, Eq. (3.4) may be written as

$$
\left[-\frac{d^{2}}{d z^{2}}+\frac{\Psi^{\prime \prime}}{\Psi}\right] \phi(z)=m^{2} \phi(z),
$$

under the assumption that $\Phi_{z}(z)=\phi(z) / \Psi(z)$, where $\Psi(z)$ $=e^{3 A(z) / 2}$. In terms of the new coordinates, $e^{A(z)}=(k \mid z$ $\left.+z_{0} \mid\right)^{-1}$ and therefore the term $\Psi^{\prime \prime} / \Psi$ behaves, near the AdS boundary, like $1 / z^{2}$ and vanishes as $z \rightarrow \infty$. As a result, this term is subdominant compared to the mass term near the singularity, a fact which, when taken into account, leads to the following solution for the $z$-dependent part of the scalar field:

$$
\Phi_{z}(z)=(k z)^{3 / 2}\left[C_{1} e^{i m z}+C_{2} e^{-i m z}\right],
$$

where $C_{1}$ and $C_{2}$ are integration constants. The scalar modes in this case are given by plane waves with a $z$-dependent amplitude and lead indeed to a vanishing flow of the fifth component of the conserved current through the boundary of the extra dimension. This may be seen by using the constraint (3.7), written in conformal coordinates

$$
\lim _{|z| \rightarrow \infty}\left[\sqrt{-\hat{g}} \hat{g}^{\mu \nu} e^{3 A} \Phi_{x}\left(\partial_{x} \Phi_{x}\right) \Phi_{z}\left(\partial_{z} \Phi_{z}\right)\right]=0,
$$

and substituting the solution (3.9). Then, the left-hand side (lhs) of the above expression turns out to be proportional to a wildly oscillating function with unit amplitude that reduces to zero.

\section{B. Vanishing warp factor at finite coordinate distance}

Another particular type of solutions that arises as a special case of the metric Ansätz (3.1) is the one that is characterized by a vanishing warp factor at finite coordinate distance, as opposed to infinite distance in the previous category. A particular example is the RS-dS-Schwarzschild black hole [1922] with line element

$$
\begin{aligned}
d s^{2}= & e^{2 A(y)}\left\{-\left(1-\frac{2 M}{r}-\Lambda r^{2}\right) d t^{2}\right. \\
& \left.+\left(1-\frac{2 M}{r}-\Lambda r^{2}\right)^{-1} d r^{2}+r^{2} d \Omega^{2}\right\}+d y^{2}
\end{aligned}
$$

and

$$
e^{A(y)}=\frac{\sinh \left[k\left(y_{0}-|y|\right)\right]}{\sinh \left(k y_{0}\right)},
$$

where here and in the following examples we shall consider $y_{0}>0$.

In the above, $\Lambda$ denotes the four-dimensional cosmological constant given by

$$
\Lambda=\frac{\kappa_{5}^{2}}{6}\left(\frac{\kappa_{5}^{2} \sigma^{2}}{6}-\left|\Lambda_{B}\right|\right),
$$

where $\sigma$ is the (positive) tension of the brane located at $y$ $=0$. For $\kappa_{5} \sigma>\sqrt{6\left|\Lambda_{B}\right|}$, the effective cosmological constant is positive, thus leading to a de Sitter four-dimensional spacetime on the brane. In this case, the warp factor vanishes at a finite coordinate distance $y=y_{0}$ from the brane, with $y_{0}$ defined by

$$
\tanh \left(k y_{0}\right)=\sqrt{\frac{6\left|\Lambda_{B}\right|}{\kappa_{5}^{2} \sigma^{2}}}
$$

The curvature-invariant quantities $R$ and $R_{A B} R^{A B}$ are found to be everywhere well-defined, however Eq. (2.6), for the square of the Riemann tensor, reduces to

$$
R_{A B C D} R^{A B C D}=40 k^{4}+\frac{48 M^{2} e^{-4 A(y)}}{r^{6}},
$$

which reveals the existence of a true singularity at $y=y_{0}$, and at the location of the black-hole singularity, $r=0$.

Another solution that falls in the same category is the one that follows if one combines the Schwarzschild-like metric tensor $\hat{g}_{\mu \nu}$ with the solution for the warp factor in the pres- 
ence of a massless bulk scalar field [23]. In this case, the five-dimensional metric tensor has the form (3.11), with $\Lambda$ $=0$, while the warp factor and the scalar field assume the form

$$
e^{4 A(y)}=a_{0}^{4} \frac{\sinh \left[\omega\left(y_{0}-|y|\right)\right]}{\sinh \left(\omega y_{0}\right)}, \quad \phi^{\prime}(y)=\frac{c a_{0}^{4}}{e^{4 A(y)}}
$$

In the above expressions, $\omega^{2}=8 \kappa_{5}^{2}\left|\Lambda_{B}\right| / 3$, while $a_{0}$ and $c$ are arbitrary constants. As in the case of the 4D de Sitter space, the warp factor vanishes at $y=y_{0}$, with $y_{0}$ being defined by

$$
\sinh \left(\omega y_{0}\right)=\sqrt{\frac{2\left|\Lambda_{B}\right|}{c^{2}}}
$$

Turning to the Riemann curvature invariant, we find the result

$$
\left(R_{A B C D}\right)^{2}=\frac{\omega^{4}}{256} \frac{\left\{207-44 \cosh \left[2 \omega\left(y_{0}-|y|\right)\right]+5 \cosh \left[4 \omega\left(y_{0}-|y|\right)\right]\right\}}{\sinh ^{4}\left[\omega\left(y_{0}-|y|\right)\right]}+\frac{48 M^{2} e^{-4 A(y)}}{r^{6}},
$$

which confirms the existence of a true singularity at $y=y_{0}$ in addition to the black-hole singularity.

A similar procedure to the one discussed in the previous subsection needs to be followed here for the resolution of the singularity problem. The boundary singularity at $y=y_{0}$ can be shielded only by the introduction of a second brane at $y$ $=L<y_{0}$. A black tube will again interpolate between the two branes, whose four-dimensional line elements will describe dS-Schwarzschild black holes, thus giving a finite size to the black-hole singularity. The monotonic profile of the warp factor in the bulk demands a negative tension for the second brane and unequal masses for the four-dimensional black holes.

\section{Nonvanishing, regular warp factor}

The last category of solutions that we are going to study corresponds to the class of five-dimensional line elements (3.1) whose warp factor is everywhere well-defined. The line element (3.11), together with Eq. (3.12), describes a four- dimensional anti-de-Sitter spacetime on the brane when $\kappa_{5} \sigma<\sqrt{6\left|\Lambda_{B}\right|}$. In that case, $\Lambda<0$ and the warp factor remains everywhere nonzero. The point $y_{0}$ now denotes the location along the extra dimension where the warp factor has a minimum. The square of the Riemann tensor is given again by Eq. (3.15), however, in this case, no divergence appears at any point along the extra dimension and the only singularity is the black-hole one.

Another solution that exhibits a similar behavior in the bulk is the one presented in Ref. [23]. The solution for the warp factor in that case was given by

$$
e^{2 A(y)}=a_{0}^{2} \cosh \left[\omega\left(|y|-y_{0}\right)\right]
$$

where $\omega^{2}=2 \kappa_{5}^{2}\left|\Lambda_{B}\right| / 3$, and the brane is at $y=0$. The metric tensor $\hat{g}_{\mu \nu}$ in the original model was the Minkowski metric tensor, $\eta_{\mu \nu}$, however the implementation of the fourdimensional Schwarzschild line element is straightforward. The square of the Riemann tensor, in this case, is found to be

$$
R_{A B C D} R^{A B C D}=\frac{\omega^{4}}{16} \frac{\left\{47+12 \cosh \left[2 \omega\left(|y|-y_{0}\right)\right]+5 \cosh \left[4 \omega\left(|y|-y_{0}\right)\right]\right\}}{\cosh ^{4}\left[\omega\left(|y|-y_{0}\right)\right]}+\frac{48 M^{2} e^{-4 A(y)}}{r^{6}},
$$

which confirms the existence of only the black-hole singularity at $r=0$.

Focusing on the second, singular term of Eqs. (3.15) and (3.20), for the $\operatorname{AdS}(\Lambda<0)$ solution (3.12) and the solution (3.19), we notice that this term, together with the black-hole singularity, vanishes exponentially fast with the distance from the brane since, for $|y| \rightarrow \infty$, we obtain

$$
\frac{48 M^{2} e^{-4 A(y)}}{r^{6}} \simeq \frac{\text { const }}{r^{6}} e^{-n \omega|y|}
$$

with $n=4$ for the AdS solution and $n=2$ for the solution (3.19), while the first term reduces to a constant in both cases. Therefore, for the above solutions, as one moves away from the brane, the black-hole singularity "decays."

The question that arises almost immediately is the behavior of gravity for those backgrounds in this case: the warp factor blows up at large distances from the brane, a fact which casts doubts on the localization of gravity. For the four-dimensional AdS spacetimes, it has been shown [21] that, although no massless zero mode exists in this case, a very light Kaluza-Klein (KK) mode can play the role of the 
massless graviton and reproduce four-dimensional gravity, provided that the effective cosmological constant, $\Lambda$, is small.

Alternatively, the localization of gravity can be achieved by means of the introduction of a second brane, as in the case of flat and dS branes. There are actually a number of options concerning the location of the second brane and thus the sign of its tension. If the second brane is placed at $y=L<y_{0}$, that is, on the lhs of the minimum, the situation resembles the one for the flat and dS branes: its tension must be negative and a black tube will interpolate between a black hole with mass $M_{1}$ on the first brane and a black hole with mass $M_{2}<M_{1}$ on the second; in addition, the black-hole singularity, due to the decrease of the value of the warp factor, will become enhanced as we move towards the second brane.

If $L=y_{0}$, the second brane necessarily has zero tension. In other words, we may use the "turn" of the warp factor in order to compactify the extra dimension without the introduction of a second brane [24], simply by identifying the two minima $y= \pm y_{0}$ on both sides of the brane. In that case, a black-hole torus would emerge from the brane, traverse the five-dimensional bulk, and return to the brane. The blackhole singularity would be most enhanced at the farthest point from the brane, that is, at the minimum $y_{0}$, while it would weaken as we moved toward the brane.

Finally, for $L>y_{0}$, that is, for any brane placed on the rhs of the minimum, the tension will always be positive, thus circumventing the necessity for the introduction of a physically unrealistic negative-tension brane. For $L=2 y_{0}$, the black tube connects two four-dimensional black holes of equal masses, $M_{1}=M_{2}$. Once we cross this critical point, $M_{2}$ will always be larger than $M_{1}$. Moreover, after this point, the black-hole singularity starts "decaying" as the singular term, in the expression of the Riemann curvature invariant, assumes smaller and smaller values compared to the one in the neighborhood of the first brane. Due to the exponential decaying behavior of this term, the black-hole singularity gets effectively "localized" for even moderate distances of the second brane from the location of the minimum $y_{0}$.

\section{THE HIGHER-DIMENSIONAL CASE}

We now turn to the study of models with two or more extra dimensions. As announced in the Introduction, we are going to consider two particular models, variants of previously derived six-dimensional brane-world solutions: the Cohen-Kaplan string solution [15] and the inhomogeneous solution of Ref. [16]. The higher-dimensional line elements will be generalized in order to include black-hole spacetime backgrounds on the brane and the analysis presented in the previous section will be extended in the particular case.

\section{A. Cohen-Kaplan-Schwarzschild solution}

Cohen and Kaplan [15] presented an interesting solution in six spacetime dimensions describing a global 3-brane modeled by a complex scalar field and zero bulk cosmological constant. The solution can be considered as a higher- dimensional generalization of the global string solutions. In the higher-dimensional version of codimension 2 , the core of the defect describes a 3-brane instead of a 1-brane. The existence of a bulk naked singularity that is mild enough to admit unitary boundary conditions results in the compactification of the extra spacetime leading to a finite radius for the transverse dimensions.

The specific form of the metric has $q$-dimensional Poincare invariance in the directions parallel to the brane and is rotationally invariant in the transverse space. Following the exact notation of Cohen and Kaplan, we write as the metric of the model

$$
d s^{2}=A^{2}(u) d \hat{s}_{q}^{2}+\gamma^{2} B^{2}(u)\left(d u^{2}+d \theta^{2}\right),
$$

where $d \hat{s}_{q}^{2}=\eta_{\mu \nu} d x^{\mu} d x^{\nu}$, and $(u, \theta)$ stand for the radial and angular coordinate, respectively, along the transverse dimensions. The metric functions $A(u)$ and $B(u)$ turn out to be

$$
A(u)=\left(\frac{u}{u_{0}}\right)^{1 / q}, \quad B(u)=\left(\frac{u_{0}}{u}\right)^{(q-1) / 2 q} e^{\left(u_{0}^{2}-u^{2}\right) / 2 u_{0}} .
$$

This metric describes the solution outside the core of the global brane defect that is located at $u \sim u_{0}$. At $u=0$, there is a naked curvature singularity at a finite proper distance from the core. Since $d \hat{s}_{q}^{2}$ is flat, we have $\hat{G}_{\mu \nu}=0$. But, as we have argued above, any solution of the vacuum equations $\hat{G}_{\mu \nu}$ $=0$ will be a solution to the higher-dimensional Einstein equations with the same functions $A(u)$ and $B(u)$. A natural solution to consider along the longitudinal directions is that of a spherical Schwarzschild black hole

$$
d \hat{s}_{\text {Schw }}^{2}=-h(r) d t^{2}+\frac{d r^{2}}{h(r)}+r^{2} d \Omega_{2}^{2},
$$

with $h(r)=1-2 M / r$. This solution has

$$
\hat{R}_{(4)}=0, \quad\left(\hat{R}_{\mu \nu \alpha \beta}\right)^{2}=\frac{48 M^{2}}{r^{6}} .
$$

In the higher-dimensional spacetime, the expression for the square of the Riemann tensor comes out to be

$$
\begin{aligned}
R_{A B C D} R^{A B C D}= & \frac{3 B^{4}(u)\left(32 u^{4}-16 u^{2} u_{0}+5 u_{0}^{2}\right)}{16 \gamma^{4} u_{0}^{5} u} \\
& +\frac{48 M^{2}}{A^{4}(u) r^{6}} .
\end{aligned}
$$

As is obvious from the second term in the above expression, the black-hole singularity, located at $r=0$, is indeed extended over the two-dimensional transverse space, however this is protected by a horizon. The same term reveals the appearance of naked singularities at the points, along the $u$ dimension, where the warp factor vanishes. However, since the only zero of the warp factor is at $u=0$, this is not going to be a new singularity but will coincide with the standard 
singularity of the original Cohen-Kaplan solution (as the first term shows, this singularity was still present even in the flat 4D spacetime limit $M \rightarrow 0$ ). The situation is similar to the five-dimensional brane-world models with a singularity in the bulk: due to the cylindrical symmetry of the transverse spacetime, the warp factor is governed by only one extra coordinate, the radial one. Any singularities appearing in the case of flat brane line elements remain unchanged when black-hole line elements are introduced, and the introduction of a 4-brane at $u=L>0$ provides the only way of shielding the naked singularity out of the causal spacetime.

In the remainder of this subsection, we will look at this naked singularity in the case of a single-brane model and establish whether its nature is changed by the introduction of the black hole on the brane or whether it can still be considered harmless as in the original work by Cohen and Kaplan. By following their analysis, we consider a more appropriate radial coordinate and move to the conformal gauge. Defining $z$ by

$$
d z=\gamma \frac{B(u)}{A(u)} d u
$$

we bring the metric to the form

$$
d s^{2}=A^{2}(z)\left(d \hat{s}_{\text {Schw }}^{2}+d z^{2}\right)+\gamma^{2} B^{2}(z) d \theta^{2} .
$$

Near the naked singularity, at $z=0$, the metric functions behave as $A(z) \rightarrow z^{2 / 3}$ and $B(z) \rightarrow z^{-1}$. In order to have a harmless naked singularity, we require the vanishing of the flow (2.13).

In [15], it was shown that unitary boundary conditions can be imposed for gravitational and scalar modes so that no conserved quantity leaks through the naked singularity. In the present case of a geometry with a black hole, the solutions are still separable, so we can introduce a scalar field

$$
\Phi(x, z, \theta)=\Phi_{x}(x) \Phi_{z}(z) e^{i n \theta} .
$$

Rewriting the $z$-dependent part of the scalar field as

$$
\Phi_{z}(z)=\frac{\varphi(z)}{\psi(z)}, \quad \psi(z) \equiv\left(\gamma A^{3} B\right)^{1 / 2}
$$

we find - not surprisingly - that it satisfies the same equation as in the Cohen-Kaplan case,

$$
\left[-\frac{d^{2}}{d z^{2}}+\frac{\psi^{\prime \prime}}{\psi}+n^{2} \frac{A^{2}}{B^{2}}\right] \varphi(z)=m^{2} \varphi(z) .
$$

The following analysis and results are identical to those of Ref. [15]: from the behavior of $A$ and $B$, as $z \rightarrow 0$, we can see that, near the singularity, the $n^{2}$ and $m^{2}$ terms are subdominant, so that all the modes behave as the zero modes in this region. These modes can be easily found by solving the above equation and their behavior, as $z \rightarrow 0$, is $\Phi_{z}^{(1)}(z) \sim 1$ and $\Phi_{z}^{(2)}(z) \sim \log z$. The condition of vanishing flow is now

$$
\lim _{z \rightarrow 0} \sqrt{-g} g^{z z} \Phi_{z}(z) \Phi_{z}^{\prime}(z)=\lim _{z \rightarrow 0} z \Phi_{z}(z) \Phi_{z}^{\prime}(z)=0,
$$

and is clearly satisfied only by $\Phi_{z}^{(1)}(z)$ thus excluding the modes of the second kind from the set of physically acceptable solutions. We thus see that there is no dramatic change in the CK solution when we introduce the intersecting black disk in the bulk. The only difference is that the $x^{\mu}$-dependent part of the scalar field must now satisfy Eq. (3.5) in a fourdimensional Schwarzschild background instead of a Minkowski one and a regular behavior should be demanded near the black-hole horizon. We therefore conclude that, in the case of the Cohen-Kaplan-Schwarzschild background, both massless and massive scalar modes, with the same trivial behavior at the vicinity of the naked singularity, are allowed to propagate in the bulk without loss of unitarity.

\section{B. Codimension-2 black branes}

In what follows, we shall consider a different class of codimension-2 metrics, based on the six-dimensional metrics presented in Ref. [16]. Unlike the previous example, the warp factor depends on both transverse coordinates $\varphi$ and $\theta$, and therefore the extra spacetime does not exhibit any cylindrical symmetry. We may write this metric as

$$
\begin{aligned}
d s^{2}= & g^{2}(\varphi) \cosh ^{2}\left[\lambda\left(\theta-\theta_{m}\right)\right] d \hat{s}_{q}^{2} \\
& +\left(d \theta^{2}+\frac{1}{\lambda^{2}} \cosh ^{2}\left[\lambda\left(\theta-\theta_{m}\right)\right] d \varphi^{2}\right),
\end{aligned}
$$

where $\lambda$ is defined as

$$
\lambda^{2}=-\frac{2 \Lambda_{B}}{q(q+1)}
$$

and it is thus associated with the radius of curvature of the $\operatorname{AdS}_{q+2}$ spacetime. On the other hand, $\theta_{m}$ gives the location of the minimum of the warp factor along the $\theta$ coordinate. The generalization with respect to [16] is that we take $q$ arbitrary while in [16] it was set equal to 4 . The total dimensionality of spacetime is thus $d=q+2$. The form of the function $g(\varphi)$ depends on the effective cosmological constant required by the metric $d \hat{s}_{q}^{2}$. Thus,

$$
\begin{aligned}
g(\varphi) & =\frac{\sinh \left\{\varphi_{0}-|\varphi|\right\}}{\sinh \left(\varphi_{0}\right)} \quad\left(\Lambda_{q}>0\right) \\
& =e^{-|\varphi|} \quad\left(\Lambda_{q}=0\right) \\
& =\frac{\cosh \left\{|\varphi|-\varphi_{0}\right\}}{\cosh \left(\varphi_{0}\right)} \quad\left(\Lambda_{q}<0\right) .
\end{aligned}
$$

Normalization is chosen so that $g(0)=1$ and we choose $\varphi_{0}$ $>0$. We note that the metrics (4.12) with $g(\varphi)$ given by the above functions and $d \hat{s}_{q}^{2}$ by the associated maximally symmetric spacetimes correspond to three different foliations of $\operatorname{AdS}_{q+2}$. The curvature radius of the $q$-dimensional metric is given by 


$$
\hat{H}^{2} \equiv \frac{2\left|\Lambda_{q}\right|}{(q-1)(q-2)}=\left(\frac{\lambda^{2}}{\sinh ^{2}\left(\varphi_{0}\right)}, 0, \frac{\lambda^{2}}{\cosh ^{2}\left(\varphi_{0}\right)}\right),
$$

for $\Lambda_{q}>0, \Lambda_{q}=0$, and $\Lambda_{q}<0$, respectively. We also note that, for $\Lambda_{q}>0$ and $\Lambda_{q}=0$, the warp factor vanishes at some point away from the brane. This signals the presence of a horizon at a finite proper distance $\left(\Lambda_{q}>0\right)$ or at an infinite proper distance $\left(\Lambda_{q}=0\right)$ from the brane.

In Ref. [16], $d \hat{s}_{q}^{2}$ corresponded to maximally symmetric line elements and a 4-brane was introduced at $\varphi=0$ by the usual procedure of imposing symmetric configurations under the transformation $\varphi \leftrightarrow-\varphi$. A positive tension brane is obtained for warp factors that decrease in the vicinity of the brane. Instead of the maximally symmetric choice for the longitudinal part of the metric, we may now proceed to consider black-hole solutions along the brane. To construct the black brane version of the above solutions, we can consider for the $q$-dimensional part of the metric the following Ansätz:

$$
d \hat{s}_{q}^{2}=-h(r) d t^{2}+\frac{d r^{2}}{h(r)}+r^{2} d \Omega_{(q-2)}^{2},
$$

with

$$
h(r)=1-\left(\frac{2 M}{r}\right)^{(q-3)}+\epsilon \hat{H}^{2} r^{2} .
$$

In this case, we have

$$
\begin{aligned}
\hat{R}_{(q)} & =-\epsilon q(q-1) \hat{H}^{2}, \\
\left(\hat{R}_{\mu \nu \alpha \beta}\right)^{2} & =\frac{4(q-1)(q-2)^{2}(q-3) M^{2(q-3)}}{r^{2(q-1)}}+2 q(q-1) \hat{H}^{4} .
\end{aligned}
$$

These metrics represent pure Schwarzschild $(\epsilon=0)$, Schwarzschild-de Sitter $(\epsilon=-1)$, and Schwarzschildanti-de Sitter $(\epsilon=1)$ black holes in a $q$-dimensional spacetime.

Not surprisingly, the introduction of the black brane gives rise to an extended naked singularity. We can see this from the expression of the square of the Riemann tensor for these $(q+2)$-dimensional metrics,

$$
\begin{aligned}
\left(R_{A B C D}\right)^{2}= & 2(q+1)(q+2) \lambda^{4} \\
& +\frac{4(q-1)(q-2)^{2}(q-3) M^{2(q-3)}}{\cosh ^{4}\left[\lambda\left(\theta-\theta_{m}\right)\right] g^{4}(\varphi) r^{2(q-1)}} .
\end{aligned}
$$

Here, we can see explicitly how the horizons become singular. Any zero of $g(\varphi)$ signals a naked singularity. For the particular solutions under consideration, $g(\varphi)$ goes to zero for $\Lambda_{q}>0$ as $\varphi \rightarrow \varphi_{0}$ and for $\Lambda_{q}=0$ as $|\varphi| \rightarrow \infty$. For $\Lambda_{q}$ $<0$, there is no new singularity since the warp factor is always different from zero. If we keep the $\theta$ coordinate fixed, then the profile of the warp factor along the $\varphi$ coordi- nate is similar to the one in the case of the five-dimensional spacetimes considered in Sec. III: for $\Lambda_{q}$ zero or positive, the higher-dimensional spacetime is characterized by two bulk singularities, one covered by a horizon, the other naked; in order to shield the second singularity and give a finite size to the first one, we need to introduce a second brane at $\varphi$ $=\varphi_{L}$, before the zero of the warp factor takes place. For $\Lambda_{q}$ negative, the warp factor along the $\varphi$ coordinate has a coshlike behavior which causes the suppression of the black-hole singularity for distances $\varphi>\varphi_{0}$, i.e., for distances moderately larger than the location of the minimum of the warp factor. In this last case, therefore, no brane needs to be introduced as the black-hole singularity rapidly decays away from the brane, while an effective compactification may take place through the mechanism of Ref. [21].

If we now allow for the $\theta$ coordinate to vary as well, then an additional "localizing" effect appears. Due to the absence of any cylindrical symmetry, as opposed to the model studied in the previous subsection, the warp factor depends on both transverse coordinates and thus an extra cosh-like function appears in the denominator of the singular term in the expression of $\left(R_{A B C D}\right)^{2}$. The $\theta$ profile of the warp factor remains the same for every value of $\Lambda_{q}$ and leads to the suppression of this singular term at distances $\theta>\theta_{m}$, independently of the form of the $\varphi$ profile. Because of the cosh-like profile along this dimension, an effective compactification may also take place as in Ref. [21].

We next consider the flow into the naked singularity for scalar field modes and check that no conserved quantity leaks through it. The form of the metric leads again to separable equations. So we can write the scalar field mode as $\Phi(x, \theta, \varphi)=\Phi_{x}(x) \Phi_{\theta}(\theta) \Phi_{\varphi}(\varphi)$. The flow vanishing condition (2.13) now leads to

$$
\lim _{\varphi \rightarrow \varphi_{0}} g^{q}(\varphi) \Phi_{\varphi}(\varphi) \Phi_{\varphi}^{\prime}(\varphi)=0
$$

Thus, we need only the explicit behavior along the $\varphi$ direction close to the singularity. The separated equations for the scalar modes along the extra spacetime are

$$
\begin{array}{r}
g^{-(q-2)} \partial_{\varphi}\left(g^{q} \partial_{\varphi} \Phi_{\varphi}\right)+\Phi_{\varphi}\left(m^{2}-k g^{2}\right)=0, \\
f^{-(q-1)} \partial_{\theta}\left(f^{(q+1)} \partial_{\theta} \Phi_{\theta}\right)+k \Phi_{\theta}=0,
\end{array}
$$

where $m^{2}$ is the mass of the modes from the 4D perspective, $k$ is a separation constant, and $f=\cosh \left[\lambda\left(\theta-\theta_{m}\right)\right]$. Unlike the Cohen-Kaplan example, near the singularity, when $g \rightarrow 0$, the massive term in the first equation is not subdominant, although the $k$ term is.

It is convenient to change the independent variable so that $d z=d \varphi / \lambda g(\varphi)$ and rescale

$$
\Phi_{z}(z)=\frac{\chi(z)}{\Psi(z)}, \quad \Psi(z)=g(z)^{(q-1) / 2},
$$


so that the equation takes the form of a quantum mechanics problem $^{3}$

$$
\left[-\frac{d^{2}}{d z^{2}}+V\right] \chi=m^{2} \chi, \quad V \equiv \frac{\Psi^{\prime \prime}}{\Psi}+k g^{2},
$$

where primes now stand for $\partial_{z}$. In the limit $g \rightarrow 0$, we can safely drop the $k$ term.

We first address the $\Lambda_{q}>0$ case where $g \propto \sinh \left(\varphi_{0}-|\varphi|\right)$ $=1 / \sinh \left(H\left(z_{0}+|z|\right)\right)$ so the limit $\varphi \rightarrow \varphi_{0}$ corresponds to $z$ $\rightarrow \infty$. Due to the reflection symmetry across the wall, we will consider only the singularity at one side of the wall. In the limit $z \rightarrow \infty$, we have

$$
-\frac{d^{2}}{d z^{2}} \chi=\left[m^{2}-\frac{(q-1)^{2}}{4} \lambda^{2}\right] \chi .
$$

An approximate solution near the singularity is given in terms of rising and falling exponentials

$$
\chi_{ \pm}(z)=e^{ \pm \Delta z}
$$

with $\Delta \equiv \sqrt{(q-1)^{2} \lambda^{2} / 4-m^{2}}$. The flow into the singularity (4.22) for these two modes is proportional to

$$
\lim _{z \rightarrow \infty} g^{(q-1)}(z) \Phi_{z}(z) \Phi_{z}^{\prime}(z)=\lim _{z \rightarrow \infty}\left[e^{2 \Delta z}, e^{-2 \Delta z}\right]
$$

for $\chi_{+}$and $\chi_{-}$, respectively. Note that there is a critical value $m_{c}^{2} \equiv(q-1)^{2} \lambda^{2} / 4$. For $0 \leqslant m^{2}<m_{c}^{2}, \Delta$ is real and it is clear that only the mode given by $\chi_{-}$is physical, leading to a vanishing flow through the singularity. For $m^{2}>m_{c}^{2}$, the modes are proportional to plane waves and the limit corresponds to a wild oscillation, leading again to a vanishing flow.

One can wonder about the existence of modes with $\mathrm{m}^{2}$ $<m_{c}^{2}$. After all, $m_{c}^{2}$ is the constant value of the potential as $z \rightarrow \infty$ and we know that, at best, we will have a single normalizable mode when $m_{c}^{2}$ is not the absolute minimum of the potential. It is natural thus to look for those modes and see whether they coincide with the vanishing flow modes. For this we have to consider the full expression of the potential for $\chi$,

$$
\begin{aligned}
V= & \frac{(q-1)^{2} \lambda^{2}}{4}+\frac{\left(q^{2}-1\right) \lambda^{2}+4 k \sinh ^{2}\left(\lambda z_{0}\right)}{4 \sinh ^{2}\left(\lambda\left(z_{0}+|z|\right)\right)} \\
& -(q-1) \lambda \operatorname{coth}\left(\lambda z_{0}\right) \delta(z) .
\end{aligned}
$$

The above is similar to the known volcano potential for a de Sitter brane in $\mathrm{AdS}_{5}$. We recover that expression for $q$

\footnotetext{
${ }^{3}$ Note that the normalization for the modes comes from the condition

$$
\sim \chi(z)^{2} d z<\infty \text {. }
$$$$
\int \sqrt{g} g^{A B} \partial_{A} \Phi \partial_{B} \Phi \supset \int \sqrt{g}[g(\varphi) \cosh (\lambda \theta)]^{-2} \hat{g}^{\mu \nu} \partial_{\mu} \phi \partial_{\nu} \phi
$$

$=4, k=0$. The $\delta$ function due to the presence of the wall supports the zero mode, for $k=0$, and then we have the continuum of KK modes with the usual gap $m^{2}>m_{c}^{2}$. Since the KK modes lie at $m^{2}>m_{c}^{2}$, they clearly satisfy the vanishing flow condition. We only have to check that the zero mode with the vanishing flow is normalizable and thus that it corresponds to the localized graviton. The zero mode with vanishing flow is approximated by $\chi(z) \sim e^{-\lambda|z|(q-1) / 2}$ as $|z| \rightarrow \infty$, so the integral for $\chi^{2}(z)$ will converge. We have thus shown that none of the normalizable modes leaks through the singularity.

In the $\Lambda_{q}=0$ case, $g(\varphi)=e^{-|\varphi|}=1 /(1+\lambda|z|)$. In the new parametrization, the singularity is approached when $z \rightarrow \infty$, and the equation for the scalar modes can be approximated by

$$
\left[-\frac{d^{2}}{d z^{2}}+\frac{\left(q^{2}-1\right) \lambda^{2}+4 k}{4 \lambda^{2}} \frac{1}{z^{2}}\right] \chi=m^{2} \chi
$$

For massless modes, close to the singularity we have

$$
\chi_{ \pm}(z) \sim(\lambda z)^{\left(1 \pm \sqrt{q^{2}+4 k / \lambda^{2}}\right) / 2},
$$

and the flow is proportional to

$$
\begin{aligned}
\lim _{z \rightarrow \infty} & g^{(q-1)}(z) \Phi_{z}(z) \Phi_{z}^{\prime}(z) \\
& \sim \lim _{z \rightarrow \infty}\left[(\lambda z)^{\sqrt{q^{2}+4 k / \lambda^{2}}},(\lambda z)^{-\sqrt{q^{2}+4 k / \lambda^{2}}}\right]
\end{aligned}
$$

for $\chi_{+}$and $\chi_{-}$, respectively. Clearly, $\chi_{-}$corresponds to the acceptable massless mode with vanishing flow through the singularity. Finally, for massive modes, we can drop the term in $1 / z^{2}$ in Eq. (4.31) and we find again plane waves

$$
\chi(z)=C_{1} e^{i m z}+C_{2} e^{-i m z},
$$

which lead to vanishing flow through the singularity and can be normalized in the usual way.

For completeness, we again give the full potential, which now has the form

$$
V=\frac{\left(q^{2}-1\right) \lambda^{2}+4 k}{4(1+\lambda|z|)^{2}}-(q-1) \lambda \delta(z) .
$$

Again, for $q=4$ and $k=0$, this reduces to the volcano potential for a flat brane in $\mathrm{AdS}_{5}$. There is thus a normalizable zero mode supported by the $\delta$ function, for $k=0$, and given by $\chi_{0} \propto(1+\lambda|z|)^{(1-q) / 2}$, and the usual continuum of KK modes. As we have shown above, all of these modes have vanishing flow. We have therefore shown that, in the present example, the vanishing flow condition does not lead to additional constraints, since it is satisfied by all normalizable modes. This suggests that the naked singularity is practically harmless from this point of view, and unitarity constraints are still satisfied by scalar modes propagating in the bulk. 


\section{CONCLUSIONS}

The objective of this paper has been the investigation of the singular properties of higher-dimensional brane-world spacetimes when a spherically symmetric Schwarzschild-like black-hole background is introduced on the brane. The appearance of old and new singularities, covered by a horizon or unshielded, has been studied by computing the corresponding curvature-invariant quantities of those spacetimes. Ways of shielding or suppressing these singularities have been discussed and the possible flow of conserved currents into the naked singularities has also been studied.

In the first part of this paper, we considered various examples of factorizable five-dimensional geometries, all of which were associated with Schwarzschild black string solutions. Brane-world models with an exponential or sinh-like warp factor were additionally plagued by the presence of a naked singularity in the bulk. In these cases, the introduction of a second brane provides the only way of shielding the bulk singularity and generating a finite-size black-tube construction. On the other hand, brane-world solutions with a cosh-like warp factor are free from the bulk singularity, and we can construct brane configurations with either a compact or noncompact extra dimension. In both cases, the black string becomes effectively localized near the brane: as the warp factor increases away from the brane, the corresponding singular term, in the expression of the square of the Riemann tensor, gets suppressed at moderate distances from the brane.

We further moved, in the second part of the paper, to consider codimension- 2 models of the same factorizable type. In particular, we considered a well-known "cylindrically symmetric" model, the Cohen-Kaplan (CK) model, and the "asymmetric" six-dimensional model of Ref. [16], both appropriately modified in order to include a black hole line element on the brane. These models admit infinitely extended black brane solutions with similar features to the Schwarzschild black-string solution. The variant of the CK model is also characterized by the same naked singularity that was present in the original version-if we want this singularity shielded, we have no other choice but to introduce a second brane. The second model accepts a variety of solutions depending on the value of the effective cosmological constant on the brane: flat and positively curved branes lead to solutions with a naked bulk singularity, while negatively curved branes are bulk-singularity-free. In the first two cases, the naked singularity can be avoided and the black brane can be given a finite size only through the introduction of a second brane. In the latter case, however, the cosh-like form of the warp factor may lead once again to the effective localization of the black brane construction in a similar way to the five-dimensional case. This "asymmetric" model is characterized by an additional localizing effect that comes from the dependence of the warp factor on both extra dimensions: another cosh-like function, present for all values of the effective cosmological constant, suppresses the singular terms in the square of the Riemann tensor as one moves parallel to the brane, and thus leads to the "decaying" of both the black hole and the bulk singularity.

Single-brane configurations may be constructed if the bulk singularity is left unshielded. In this case, it is the singularity itself that terminates the extra dimension and gives a finite size to the black string or black brane. As suggested in the original analysis by Cohen and Kaplan, these types of singularities could be considered harmless if no flow of conserved quantities takes place through them. By considering an auxiliary bulk scalar field to realize a conserved current associated with the spacetime symmetries, we have studied a variety of models-five-, six- or higher-dimensional-and we have showed that it is always possible to choose appropriate particle modes in order to satisfy the constraint of vanishing flow. Attempts to construct brane-world models in five dimensions, where the naked singularity was used in the above way, resulted in solutions [25] that had either a severe, hidden fine-tuning $[26,23]$ or a badly defined effective fourdimensional theory [27] (see also [28]). A particular class of five-dimensional models that was studied here, the one with a bulk scalar field, falls in this category and therefore the shielding of the naked singularity is imperative-the introduction of the black-hole line element on the brane, in this case, did not affect the naked singularity, which preexisted. However, we have also considered alternative models, both five- and higher-dimensional ones, where the bulk singularity was induced merely by the introduction of the black hole on the brane while the bulk itself was everywhere well-defined. In these cases, it remains to be seen whether the ill-defined behavior, encountered in some five-dimensional models, also persists here or whether it can be ameliorated in the framework of more complex brane-world models.

\section{ACKNOWLEDGMENT}

I.O. and K.T. acknowledge the financial support of the EU RTN contract No. HPRN-CT-2000-00152.
[1] I. Antoniadis, Phys. Lett. B 246, 377 (1990); N. ArkaniHamed, S. Dimopoulos, and G. R. Dvali, ibid. 429, 263 (1998); I. Antoniadis, N. Arkani-Hamed, S. Dimopoulos, and G. R. Dvali, ibid. 436, 257 (1998).

[2] L. Randall and R. Sundrum, Phys. Rev. Lett. 83, 3370 (1999).

[3] L. Randall and R. Sundrum, Phys. Rev. Lett. 83, 4690 (1999).

[4] J. Lykken and L. J. Randall, J. High Energy Phys. 06, 014 (2000); K. Skenderis and P. K. Townsend, Phys. Lett. B 468, 46 (1999); K. Behrndt and M. Cvetic, ibid. 475, 253 (2000); A.
Chamblin and G. W. Gibbons, Phys. Rev. Lett. 84, 1090 (2000); O. DeWolfe, D. Z. Freedman, S. S. Gubser, and A. Karch, Phys. Rev. D 62, 046008 (2000); C. Csaki, J. Erlich, T. J. Hollowood, and Y. Shirman, Nucl. Phys. B581, 309 (2000); S. B. Giddings, E. Katz, and L. J. Randall, J. High Energy Phys. 03, 023 (2000); H. Kudoh and T. Tanaka, Phys. Rev. D 64, 084022 (2001); A. Kehagias and K. Tamvakis, hep-th/0205009.

[5] A. Chamblin, S. W. Hawking, and H. S. Reall, Phys. Rev. D 
61, 065007 (2000).

[6] I. Giannakis and H. C. Ren, Phys. Rev. D 63, 024001 (2001).

[7] R. Gregory and R. Laflamme, Phys. Rev. Lett. 70, 2837 (1993).

[8] R. Emparan, G. T. Horowitz, and R. C. Myers, J. High Energy Phys. 01, 007 (2000); J. Garriga and M. Sasaki, Phys. Rev. D 62, 043523 (2000); A. Chamblin, C. Csaki, J. Erlich, and T. J. Hollowood, ibid. 62, 044012 (2000); N. Dadhich, R. Maartens, P. Papadopoulos, and V. Rezania, Phys. Lett. B 487, 1 (2000); S. Nojiri, O. Obregon, S. D. Odintsov, and S. Ogushi, Phys. Rev. D 62, 064017 (2000); A. Chamblin, H. S. Reall, H. A. Shinkai, and T. Shiromizu, ibid. 63, 064015 (2001); I. Giannakis and H. C. Ren, ibid. 63, 125017 (2001); 64, 065015 (2001); M. Bruni, C. Germani, and R. Maartens, Phys. Rev. Lett. 87, 231302 (2001); M. Rogatko, Phys. Rev. D 64, 064014 (2001); I. Giannakis and H. C. Ren, Phys. Lett. B 528, 133 (2002); R. Casadio, A. Fabbri, and L. Mazzacurati, Phys. Rev. D 65, 084040 (2002); S. I. Vacaru and D. Singleton, Class. Quantum Grav. 19, 3583 (2002); R. Emparan, A. Fabbri, and N. Kaloper, J. High Energy Phys. 08, 043 (2002); G. Kofinas, E. Papantonopoulos, and I. Pappa, hep-th/0112019; R. Neves and C. Vaz, hep-th/0207173.

[9] C. Gomez, B. Janssen, and P. J. Silva, J. High Energy Phys. 04, 027 (2000); A. Kamenshchik, U. Moschella, and V. Pasquier, Phys. Lett. B 487, 7 (2000); D. Youm, ibid. 515, 170 (2001); Mod. Phys. Lett. A 16, 1703 (2001); C. Grojean, F. Quevedo, G. Tasinato, and I. Zavala C., J. High Energy Phys. 08, 005 (2001); S. Nojiri, S. D. Odintsov, and S. Ogushi, Int. J. Mod. Phys. A 16, 5085 (2001); R. G. Cai and Y. Z. Zhang, Phys. Rev. D 64, 104015 (2001); D. Birmingham and M. Rinaldi, Mod. Phys. Lett. A 16, 1887 (2001).

[10] H. Lu and C. N. Pope, Nucl. Phys. B598, 492 (2001); C. V. Johnson and R. C. Myers, Phys. Rev. D 64, 106002 (2001); S. S. Gubser, A. A. Tseytlin, and M. S. Volkov, J. High Energy Phys. 09, 017 (2001).

[11] H. S. Reall, Phys. Rev. D 64, 044005 (2001); G. T. Horowitz and K. Maeda, Phys. Rev. Lett. 87, 131301 (2001); J. P. Gregory and S. F. Ross, Phys. Rev. D 64, 124006 (2001).

[12] M. R. Mbonye, Phys. Rev. D 60, 124007 (1999); S. W. Hawking and H. S. Reall, ibid. 61, 024014 (2000); P. Kraus, J. High Energy Phys. 12, 011 (1999); A. M. Awad and C. V. Johnson, Phys. Rev. D 63, 124023 (2001); C. Cadeau and E. Woolgar, Class. Quantum Grav. 18, 527 (2001); R. Emparan and H. S.
Reall, Phys. Rev. Lett. 88, 101101 (2002); U. H. Danielsson, J. High Energy Phys. 03, 020 (2002).

[13] P. C. Argyres, S. Dimopoulos, and J. March-Russell, Phys. Lett. B 441, 96 (1998); R. Emparan, G. T. Horowitz, and R. C. Myers, Phys. Rev. Lett. 85, 499 (2000); R. Casadio and B. Harms, Phys. Lett. B 487, 209 (2000); Phys. Rev. D 64, 024016 (2001); F. C. Adams, G. L. Kane, M. Mbonye, and M. J. Perry, Int. J. Mod. Phys. A 16, 2399 (2001); S. B. Giddings and S. Thomas, Phys. Rev. D 65, 056010 (2002); S. Dimopoulos and G. Landsberg, Phys. Rev. Lett. 87, 161602 (2001); M. B. Voloshin, Phys. Lett. B 518, 137 (2001); Kingman Cheung, Phys. Rev. Lett. 88, 221602 (2002); Phys. Rev. D 66, 036007 (2002); P. Kanti and J. March-Russell, ibid. 66, 024023 (2002); E.-J. Ahn, M. Cavaglia, and A. Olinto, hep-th/020142; A. Chamblin and G. C. Nayak, hep-ph/0206060.

[14] P. Kanti and K. Tamvakis, Phys. Rev. D 65, 084010 (2002).

[15] A. G. Cohen and D. B. Kaplan, Phys. Lett. B 470, 52 (1999).

[16] P. Kanti, R. Madden, and K. A. Olive, Phys. Rev. D 64, 044021 (2001)

[17] I. Olasagasti and A. Vilenkin, Phys. Rev. D 62, 044014 (2000).

[18] I. Olasagasti, Phys. Rev. D 63, 124016 (2001).

[19] N. Kaloper, Phys. Rev. D 60, 123506 (1999).

[20] H. B. Kim and H. D. Kim, Phys. Rev. D 61, 064003 (2000).

[21] A. Karch and L. Randall, J. High Energy Phys. 05, 008 (2001); I. I. Kogan, S. Mouslopoulos, and A. Papazoglou, Phys. Lett. B 501, 140 (2001); A. Miemiec, Fortschr. Phys. 49, 747 (2001); M. D. Schwartz, Phys. Lett. B 502, 223 (2001); I. Oda, ibid. 508, 96 (2001); Phys. Rev. D 64, 026002 (2001).

[22] T. Hirayama and G. Kang, Phys. Rev. D 64, 064010 (2001).

[23] P. Kanti, K. A. Olive, and M. Pospelov, Phys. Lett. B 481, 386 (2000).

[24] P. Kanti, I. I. Kogan, K. A. Olive, and M. Pospelov, Phys. Lett. B 468, 31 (1999); Phys. Rev. D 61, 106004 (2000).

[25] N. Arkani-Hamed, S. Dimopoulos, N. Kaloper, and R. Sundrum, Phys. Lett. B 480, 193 (2000); S. Kachru, M. Schulz, and E. Silverstein, Phys. Rev. D 62, 045021 (2000).

[26] S. Förste, Z. Lalak, S. Lavignac, and H. P. Nilles, Phys. Lett. B 481, 360 (2000); S. P. de Alwis, Nucl. Phys. B597, 263 (2001); C. Csaki, J. Erlich, C. Grojean, and T. J. Hollowood, ibid. B584, 359 (2000).

[27] C. Charmousis, Class. Quantum Grav. 19, 83 (2002); S. C. Davis, J. High Energy Phys. 03, 058 (2002).

[28] J. M. Cline and H. Firouzjahi, Phys. Rev. D 65, 043501 (2002). 